\title{
Optimizing density down-ramp injection for beam-driven plasma wakefield accelerators
}

\author{
A. Martinez de la Ossa, ${ }^{1, *}$ Z. Hu, ${ }^{2}$ M. J. V. Streeter, ${ }^{2}$ T. J. Mehrling, ${ }^{2}$ \\ O. Kononenko, ${ }^{2}$ B. Sheeran, ${ }^{2}$ and J. Osterhoff ${ }^{2}$ \\ ${ }^{1}$ Institut für Experimentalphysik, Universität Hamburg, 22761 Hamburg, Germany \\ ${ }^{2}$ Deutsches Elektronen-Synchrotron DESY, D-22607 Hamburg, Germany
}

(Received 8 June 2017; published 6 September 2017)

\begin{abstract}
Density down-ramp (DDR) injection is a promising concept in beam-driven plasma wakefield accelerators for the generation of high-quality witness beams. We review and complement the theoretical principles of the method and employ particle-in-cell (PIC) simulations in order to determine constrains on the geometry of the density ramp and the current of the drive beam, regarding the applicability of DDR injection. Furthermore, PIC simulations are utilized to find optimized conditions for the production of high-quality beams. We find and explain the intriguing result that the injection of an increased charge by means of a steepened ramp favors the generation of beams with lower emittance. Exploiting this fact enables the production of beams with high charge $(\sim 140 \mathrm{pC})$, low normalized emittance $(\sim 200 \mathrm{~nm})$ and low uncorrelated energy spread $(0.3 \%)$ in sufficiently steep ramps even for drive beams with moderate peak current $(\sim 2.5 \mathrm{kA})$.
\end{abstract}

DOI: 10.1103/PhysRevAccelBeams.20.091301

\section{INTRODUCTION}

Beam-driven plasma wakefield accelerators (PWFAs) $[1,2]$ can generate and sustain accelerating gradients in excess of $\sim 10 \mathrm{GV} / \mathrm{m}$ over meter-scale distances. Harnessing such extreme fields for the production of multi-GeV energy, high-brightness electron beams can enable a new generation of accelerators, capable of compactly driving applications, e.g., in particle physics, medicine, or materials science, at considerably reduced costs. The first successful experiments employing electron beams as plasma wakefield drivers have been conducted over the last 15 years at SLAC. The energy doubling of $42 \mathrm{GeV}$ electrons in the tail of an electron beam [3] in a meter-long plasma cell, and the demonstration of efficient energy gain using a drive-bunch/trailing-bunch scheme in a $36 \mathrm{~cm}$-long plasma source [4] constitute landmark results in PWFAs. Besides the extreme accelerating fields demonstrated in PWFAs, improved control over the injection of beams is a necessary step towards the production of beams of sufficient quality (low emittance and energy spread) for applications such as free-electron lasers or future compact linear colliders.

In recent years, a number of novel beam injection techniques for PWFAs have been proposed in order to achieve the required control over the accelerated bunches.

*alberto.martinez.de.la.ossa@desy.de

Published by the American Physical Society under the terms of the Creative Commons Attribution 4.0 International license. Further distribution of this work must maintain attribution to the author(s) and the published article's title, journal citation, and DOI.
Trapping of electrons originated from beam field-ionization in PWFAs was first observed in an experiment at FACET [5]. Since then, more elaborate techniques based on ionization have been proposed that employ either assistive lasers [6,7] or the wakefields themselves [8] to achieve improved control over the injected beam parameters, and therefore, higher beam quality. Notwithstanding the promising capabilities of ionization-based injection techniques, their implementation is limited to high-current drivers ( $\gtrsim 5 \mathrm{kA}$ ) [9], that generate plasma waves in a strong blowout regime [10,11].

Another promising approach consists on injecting electrons from the background plasma by means of controlled wavebreaking during a plasma density down-ramp (DDR) transition $[12,13]$. The conditions for trapping in terms of the required beam current are relaxed for this scheme. This is due to the reduction of the phase velocity of the wakefields during the DDR, which allows to trap background plasma electrons even when employing lower current drivers. While DDR injection has been successfully applied to laser-driven plasma wakefield accelerators (LWFA) in multiple experiments [14-17], only recently it has been possible to implement this injection technique in PWFA (FACET E-210). Further experiments on PWFA with DDR injection are foreseen to be conducted in FLASHForward [18], a new facility under construction at DESY which will make use of the electron beams from the FLASH accelerator to drive novel PWFA experiments. Particle-in-cell (PIC) simulations, considering FLASHForward-like parameters, have shown that it is possible to generate relatively long ( $\sim 40$ fs FWHM) and low emittance ( $200 \mathrm{~nm})$ beams in PWFAs by employing sufficiently steep linear DDRs [19] and relatively lowcurrent driver beams $(\sim 2.5 \mathrm{kA})$. Suitable conditions for the 
plasma DDRs could be achieved by means of gas profile tailoring [20] or optical-ionization methods [21]. The latter allows for the generation of localized plasma density spikes, and promise to deliver sufficiently steep density gradients for DDR injection, even when using relatively low-current drivers.

In this work, we review the theoretical principles of the DDR injection technique for PWFAs in order to determine the limits of its applicability when using realistic DDR profiles (Sec. II). Based on these findings, we analyze in detail the properties of the produced bunches by means of PIC simulations with the code OSIRIS [22]. The parameters of the ramp (length and peak density) are varied in order to find optimal conditions for the production of highquality beams (Sec. III). We find that shorter ramps lead to an increase of the trapped charge, which in turn, contributes to substantially reduce the emittance and the energy spread of the injected beams. The underlying physics of this intriguing correlation between higher injected charge in steeper ramps and reduced emittance is explained theoretically (Sec. IV). Exploiting this fact, we demonstrate that it is possible to use the DDR technique for the production and acceleration of high-quality beams (high charge, low emittance, and low energy spread) in the wakefields created by $2.5 \mathrm{kA}$ electron drivers, when employing DDRs shorter than the plasma wavelength at the plateau density.

\section{DENSITY DOWN-RAMP INJECTION MODEL}

PWFAs [1,2] use relativistic charged particle beams that are sufficiently dense to significantly displace plasma electrons by means of their space-charge fields. As the beam passes, the displaced plasma electrons are attracted back by the excess of positive charge, and oscillate around an equilibrium position, generating in this way a plasma density perturbation which propagates at the velocity of the beam. For small displacements, these oscillations are harmonic at a frequency given by $\omega_{p}=\sqrt{n e^{2} / m \epsilon_{0}}$ and a wave number of $k_{p}=\omega_{p} / c$, where $n$ is the electron density of the plasma, $\epsilon_{0}$ is the vacuum permittivity, $c$ is the speed of light, and $m$ and $e$ are the electron mass and charge, respectively.

\section{A. Phase velocity and density ramps}

We start defining the phase-position of an electron in a plasma wave by $\chi \equiv k_{p}\left(z-\beta_{b} c t\right)$, where $z$ is the longitudinal position of the electron and $\beta_{b}$ is the velocity of the drive-beam normalized to the speed of light. If the plasma density profile $n(z)$ varies as the position of the electron $z$ changes, the phase-position of an electron with (normalized) longitudinal velocity $\beta_{z}$ changes accordingly with

$$
\frac{d \chi}{d z}=\frac{1}{2} \frac{\chi}{n} \frac{d n}{d z}+k_{p}\left(1-\frac{\beta_{b}}{\beta_{z}}\right) .
$$

In the case of a constant plasma density, the first term in Eq. (1) is identically 0 , and the change of the phase-position of an electron depends only on its own velocity, relative to the velocity of the plasma wave, i.e., the velocity of the drive-beam. However, during a density transition, there is an additional phase-shift caused by the change of the density, that is, due to the change in the frequency of the plasma response. The phase-velocity of the plasma wave at the location of the electron is obtained from Eq. (1) with $d \chi / d z=0$

$$
\beta_{\mathrm{ph}}=\beta_{b}\left(1+\frac{\chi}{2 \tilde{n}^{3 / 2}} \frac{1}{k_{p}^{0}} \frac{\mathrm{d} \tilde{n}}{\mathrm{~d} z}\right)^{-1}
$$

where $\tilde{n}=n / n^{0}$ is the local plasma density normalized to a reference plasma density $n^{0}$, and $k_{p}^{0}$ the wave number for $n^{0}$. During a plasma density down-ramp (DDR) $(\mathrm{d} \tilde{n} / \mathrm{d} z<0)$, the phase velocity behind the driver is reduced $\left(\beta_{\mathrm{ph}}<\beta_{b}\right)$. This effect is more pronounced the larger the distance behind the driver, the steeper the density gradient and the lower the density at which the plasma transition happens.

\section{B. Trapping condition}

By using Eq. (2) in Eq. (1), we find the following expression for the phase shift of a plasma electron during a plasma density transition

$$
\frac{\mathrm{d} \chi}{\mathrm{d} z}=k_{p} \beta_{b}\left(\frac{1}{\beta_{\mathrm{ph}}}-\frac{1}{\beta_{z}}\right) .
$$

Plasma electrons at a certain phase-position $\chi$ and with a certain velocity $\beta_{z}$, can be locked or advanced in phase whenever $\beta_{z} \geq \beta_{\mathrm{ph}}$. This is the main idea behind the DDR injection method: A plasma electron can become trapped in the plasma wake if it propagates equal to or faster than $\beta_{\mathrm{ph}}$, at the phase-position of the electron. During the first half of the plasma oscillation, the electrons are accelerated backwards, while in the second half, they are accelerated in forward direction. Therefore, the electrons reach the maximum longitudinal velocity $\beta_{z, \max }$ at the end of each oscillation, i.e., $\chi_{N}=\chi_{1}-k_{p} \lambda_{w}(N-1)$ where $N$ specifies the $N$ th spatial period of the wake, $\lambda_{w}$, and $\chi_{1}$ represents the phase-position at the end of the first accelerating bucket. At these points, the longitudinal electric fields change from negative to positive values, delimiting the different oscillation periods of the plasma wake. In case of a linear wakefield excitation regime $\chi_{1}=-3 \pi / 2$ and $k_{p} \lambda_{w}=2 \pi$. In the non-linear regime, both $\chi_{1}$ and $k_{p} \lambda_{w}$ generally depend on the driver parameters and the plasma density value. However, the dominating effect leading to trapping in DDRs is the phase velocity reduction and the effects derived from a density dependent $k_{p} \lambda_{w}$ can be neglected, for the cases considered in this work. Hence, if $\beta_{z}\left(\chi_{N}\right)>$ $\beta_{\mathrm{ph}}\left(\chi_{N}\right)$, the plasma electrons do not leave the accelerating 
region of the $N$ th period of the wake, where they quickly gain more velocity up to near the speed of light $\left(\beta_{z} \simeq 1\right)$. We call these trapped electrons.

\section{Rephasing of trapped electrons}

The phase shift of a trapped electron (with $\beta_{z} \rightarrow 1$ ), caused by further changes in density, is obtained by integrating Eq. (1) from the moment of the injection, at $z_{i}$, until an arbitrary point $z$ downstream the ramp (for $\left.\beta_{b} \approx 1\right)$ :

$$
\chi-\chi_{i}=\chi_{i}\left(\sqrt{\frac{n}{n_{i}}}-1\right)
$$

Here $\chi\left(\chi_{i}\right)$ is the final (initial) phase position, and $n\left(n_{i}\right)$ is the final (initial) density value. Equation (4) shows that trapped electrons move forward $\left(\chi>\chi_{i}\right)$ with respect to the plasma wake when $n<n_{i}$, and that the phase shift is proportional to the initial phase position $\chi_{i}$. Electron trapping in later periods of the plasma wake is generally easier due to the stronger reduction of the phase velocity [Eq. (2)]. However, electrons injected in later periods will also experience a larger phase shift [Eq. (4)]. Because we would like the electrons to remain in the acceleration region of the $N$ th period of the wake, the maximum phase shift $\left(\Delta \chi_{\max }\right)$ should not be greater than approximately half the length of the plasma oscillation bucket $\left(k_{p} \lambda_{w} / 2\right)$. This requirement sets a limit for the maximum density value at which injection needs to happen

$$
\frac{n_{i}}{n}<\left(\frac{1}{1+\Delta \chi_{\max } / \chi_{N}}\right)^{2}
$$

with $\Delta \chi_{\max } / \chi_{N} \simeq-1 / 2 N$. The condition is most relaxed for the first plasma period $(N=1)$, for which $\frac{n_{i}}{n}<4$. Eq. (4) can also be used to estimate the length of the injected bunches. If we call $\tilde{n}_{i}\left(\tilde{n}_{f}\right)$ to the first (last) density value at which trapping happens, the total length of the trapped bunch can be estimated by

$$
k_{p}^{0} L=\frac{\chi_{i}}{\sqrt{\tilde{n}_{i}}}-\frac{\chi_{f}}{\sqrt{\tilde{n}_{f}}},
$$

where $\chi_{i}\left(\chi_{f}\right)$ is the initial phase position of the first (last) trapped electron. (this expression can be further simplify when $\left.\chi_{i} \approx \chi_{f} \approx \chi_{N}\right)$.

\section{Gaussian ramps}

In the following we will make use of Gaussian DDRs, as they represent more realistically than linear ramps the kind of density transitions experimentally achievable [23]:

$$
\tilde{n}(z)=1+\left(\tilde{n}_{\text {top }}-1\right) \exp \left(-\frac{z^{2}}{2 \sigma_{l}^{2}}\right)
$$
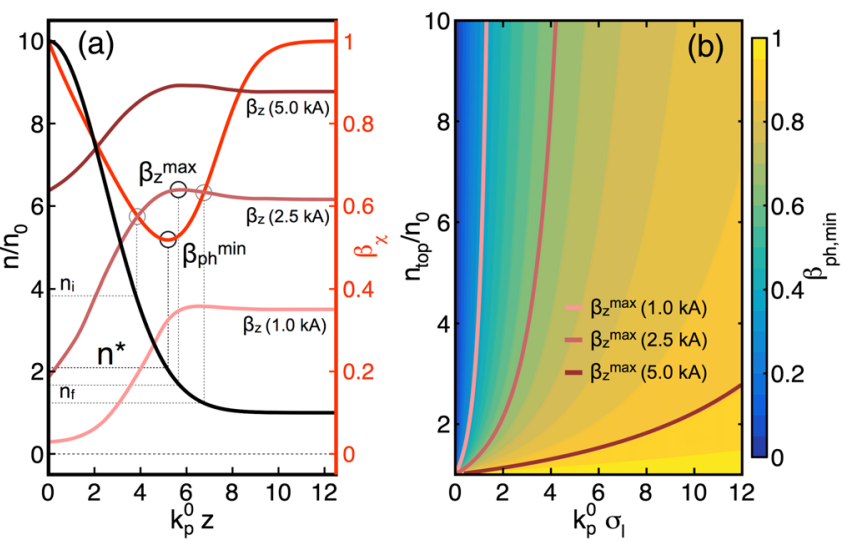

FIG. 1. (a) Gaussian density down-ramp (black) (with $\tilde{n}_{\text {top }}=$ 10 and $k_{p}^{0} \sigma_{l}=2.5$ ) and phase velocity of the plasma wave (red) at the phase-position $\chi=-2 \pi$, as a function of the distance with respect to the top of the ramp. The maximum velocity of the plasma electrons driven by Gaussian electron beams with 1, 2.5 and $5.0 \mathrm{kA}$ of peak current, as a function of the plasma density is also shown for comparison. (b) Minimum phase velocity at $\chi=-2 \pi$ as a function of the ramp parameters $\tilde{n}_{\text {top }}$ and $k_{p}^{0} \sigma_{l}$ (color map). The maximum velocity of the plasma electron for 1 , 2.5 and $5.0 \mathrm{kA}$ electron drivers is also shown as red contours.

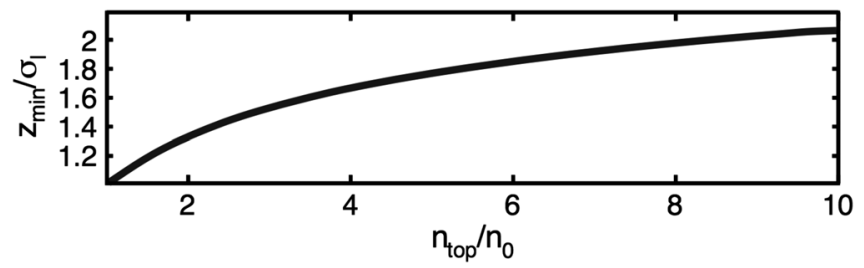

FIG. 2. Position of the minimum phase velocity $z_{\min } / \sigma_{l}$ as a function of $\tilde{n}_{\text {top }}$.

for $z \geq 0$, where $\tilde{n}_{\text {top }}$ is the top density normalized to the plateau value and $\sigma_{l}$ is the characteristic decay length of the ramp. In Fig. 1(a) we show as a reference $\beta_{\mathrm{ph}}$ at $\chi=-2 \pi$, calculated from Eq. (2), for a Gaussian DDR with $\tilde{n}_{\text {top }}=10$ and $\tilde{\sigma}_{l} \equiv k_{p}^{0} \sigma_{l}=2.5$. Using Eq. (7) in Eq. (2), we find analytically that $z_{\min } / \sigma_{l}$, the longitudinal position of the minimum phase velocity (normalized to $\sigma_{l}$ ), only depends on the value of the density at the top of the ramp $\tilde{n}_{\text {top }}$ (cf. Fig. 2). For the case shown in Fig. 1(a), we have that $z_{\text {min }} \approx 2.1 \sigma_{l}$ and $\beta_{\text {ph,min }} \approx 0.52$. Figure $1(\mathrm{~b})$ shows $\beta_{\mathrm{ph}, \min }$ as a function of the ramp parameters $\tilde{n}_{\text {top }}$ and $\tilde{\sigma}_{l}$.

\section{E. Electron velocity and wakefield potential}

In order to provide an estimation of the actual velocity of the electrons in an electron-beam driven plasma wake (here $\beta_{b} \approx 1$ ), we make use of a constant of motion [24] that holds under the quasistatic approximation (QSA) [25], i.e., $\mathcal{K}=\gamma\left(1-\beta_{z}\right)-\psi$, with $\gamma=1 / \sqrt{1-\boldsymbol{\beta}^{2}}$ the Lorentz factor of the electrons and $\psi=\frac{e}{m c^{2}}\left(\Phi-c A_{z}\right)$ the 
normalized wakefield-potential, related to the longitudinal and transverse wakefields by $E_{z} / E_{0}=-\partial_{z} \psi$ and $\left(E_{r}-c B_{\phi}\right) / E_{0}=-\partial_{r} \psi$, respectively. Assuming that initially the plasma electrons are at rest and $\psi=0$ prior the arrival of the driver, the constant of motion is $\mathcal{K}=1$. Under these conditions the longitudinal velocity of a plasma electron reads

$$
\beta_{z}=\frac{1-(1+\psi)^{2} \epsilon\left(\psi, \beta_{x}\right)}{1+(1+\psi)^{2}}
$$

where $\epsilon\left(\psi, \beta_{x}\right)=\sqrt{1-\beta_{x}^{2}\left(1+(1+\psi)^{2}\right) /(1+\psi)^{2}}$. Further assuming that the longitudinal component of the electron velocity dominates $\gamma$ (i.e., $\beta_{x} \ll \beta_{z}$ ) we find that $\epsilon \approx 1$ and then $\beta_{z}$ is defined in terms of $\psi$ only. The quasistatic approximation assumes that the fields and currents are frozen, or quasistatic, during the plasma evolution in the comoving frame, i.e., $\partial_{t} \simeq-\partial_{\zeta}$ for these quantities. Although this condition will be broken during the process of injection along a density down-ramp, Eq. (8) can still provide an estimation of the longitudinal velocity of the plasma electrons $\left(\beta_{z}\right)$ by knowing the value of the potential $(\psi)$ at the phase of interest, and assuming that $\beta_{x}^{2} \ll 1$. For this reason, we discuss in the following the structure of $\psi$ in beam-driven plasma wakefield accelerators.

Analytical solutions for $\psi$ can be found in the linear regime of PWFAs by linearizing the Maxwell equations and the equations of motion for the plasma electrons. However, the assumptions for the linear regime hold only when $|\psi| \ll 1$, and therefore, $\left|\beta_{z}\right| \ll 1$. This means that, in the linear regime, the geometrical requirements for the DDR in order to reduce the phase velocity below the maximum velocity of the plasma electrons are strongly demanding, and the applicability of the method is limited to highly steep ramps. In contrast, when the plasma is operated in blowout regime, electrons can acquire high longitudinal velocities after every oscillation, and therefore, the requirements for the DDR can be largely relaxed.

High-current $\left(I_{b} \gtrsim 1 \mathrm{kA}\right)$ and narrow $\left(k_{p} \sigma_{r} \lesssim 1\right)$ electron drivers blow out all the plasma electrons from their propagation path, creating an ion cavity with no plasma electrons inside. As can be seen in PIC simulations (Fig. 3(a)), this ion-cavity (or blowout) is delimited by a sheath of plasma electrons, which accumulate at a certain distance from the propagation axis. The phenomenological models for the blowout regime $[11,26,27]$ connect $E_{z}$ and $\psi$ [Figs. 3(b) and (c), respectively] inside the ion-cavity with the dynamics of the plasma electrons in the plasma sheath. However these models fail to provide an accurate value for $\psi$ precisely in the vertex of the blowout, where $\psi$ reaches its minimum, the plasma electrons are the fastest, and from where is easiest to have injection by means of a density down-ramp. For this reason, we have used 3D full PIC simulations, employing the code OSIRIS [22], to explore the wakefields driven by high-current electron beams. They

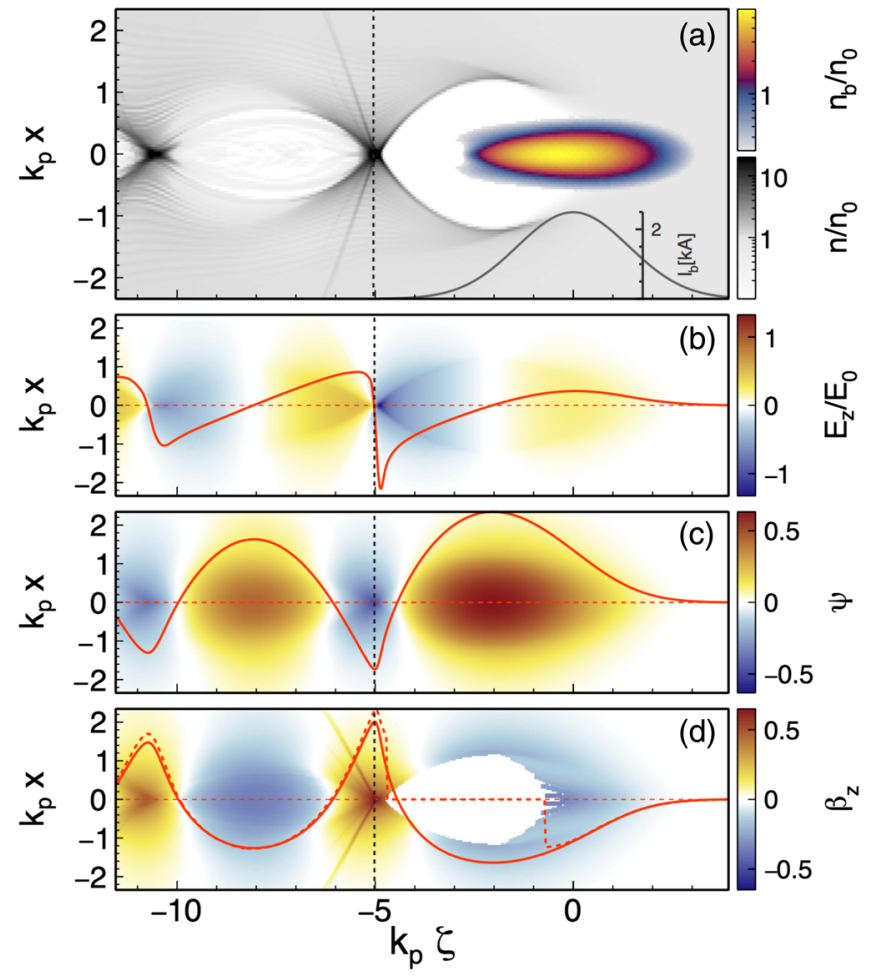

FIG. 3. OSIRIS 3D simulation of an axially symmetric Gaussian electron beam with peak current $I_{b}^{0}=2.5 \mathrm{kA}$, transverse rms size $k_{p} \sigma_{r}=0.3$, and longitudinal rms length $k_{p} \sigma_{z}=\sqrt{2}$. The driver beam operates an uniform plasma at the resonant density. (a) Plasma $(n)$ and beam $\left(n_{b}\right)$ electron density. (b) Longitudinal electric field $\left(E_{z}\right)$. (c) Wakefield potential $(\psi)$. (d) Longitudinal electron velocity $\left(\beta_{z}\right)$. The solid curve in (d) represents $\beta_{z}$ as calculated from $\psi$ according to Eq. (8) (with $\epsilon=1$ ), while the dashed curve shows the value of $\beta_{z}$ retrieved from the simulation.

consist of a series of simulations with identical beam parameters apart from the peak current, which ranges 1, 2.5 , and $5 \mathrm{kA}$. These electron beams are sent through a long Gaussian density down-ramp $\left(k_{p}^{0} \sigma_{l}=68.5\right)$ in order to probe the wakefield structure at different densities along the ramp, while minimizing the electron re-phasing effect due to density variations [cf. Eq. (1)]. In addition, the beams were initialized to an energy of $100 \mathrm{GeV}$ in order to avoid the effect of a changing beam radius due to the betatron oscillation of the envelope of the beam (rigid beam approximation). The geometry of the Gaussian ramp is such that the density value at $z=2 \sigma_{l}$ complies with the resonant density in the linear regime [28], i.e. $k_{p}^{*} \sigma_{z}=\sqrt{2}$, with $k_{p}^{*}$ the plasma oscillation wave number corresponding to the resonant density $n^{*}$. Therefore, by construction, $\tilde{n}^{*}=$ $1+\left(\tilde{n}_{\text {top }}-1\right) / \exp (2)$ in this series of simulations. Figure 4 (bottom) shows the evolution of $\psi$ in the propagation axis [red curve in Fig. 3(c)] as a function of the propagation distance, for the simulation with $I_{b}^{0}=2.5 \mathrm{kA}$. As can be seen in Fig. 3 the zero-crossings of $E_{z}$ correspond to the phase position of the $\psi$ extrema. We 


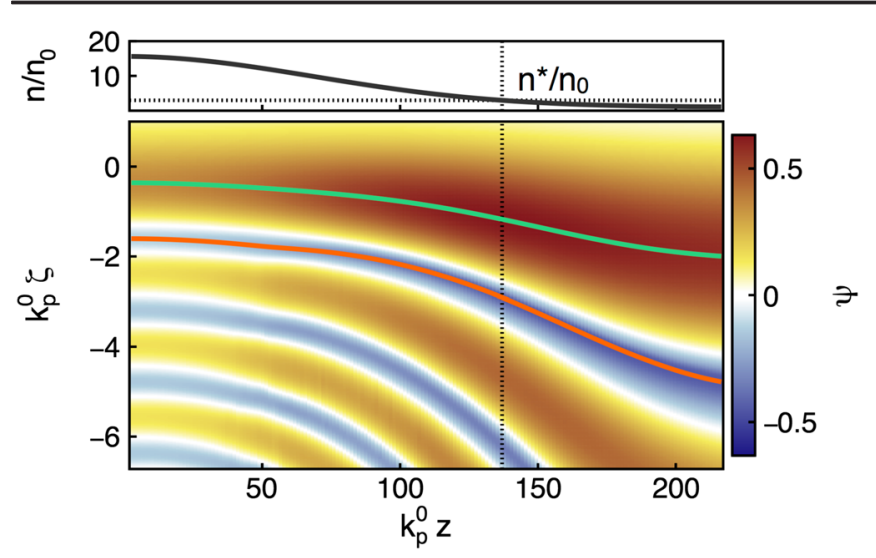

FIG. 4. Evolution of the on-axis wakefield potential (bottom) along a Gaussian DDR $\left(k_{p}^{0} \sigma_{l}=68.5\right)$ (top) for a beam with $I_{b}^{0}=2.5 \mathrm{kA}$. $\quad \tilde{n}_{\text {top }}=15.63$ in the simulation and $\tilde{n}^{*}=1+$ $\left(\tilde{n}_{\text {top }}-1\right) / \exp (2)$. The vertical dotted line marks the $z=2 \sigma_{l}$ position at which $n=n^{*}$, with $n^{*}$ the resonant density. The positions of $\psi_{\max }$ and $\psi_{\min }$ are drawn in green and orange, respectively.

track these positions to obtain $\psi_{\min }$ and $\psi_{\max }$ as a function of $z$ [Fig. 4 (bottom)], and using $\tilde{n}(z)$ [Fig. 4 (top)], we obtain the dependence of these quantities on the plasma density. Figure 5(a) shows $\psi_{\min }$ and $\psi_{\max }$ as a function of $\tilde{n}$ for the three simulations with $I_{b}^{0}=1.0,2.5$, and $5.0 \mathrm{kA}$.

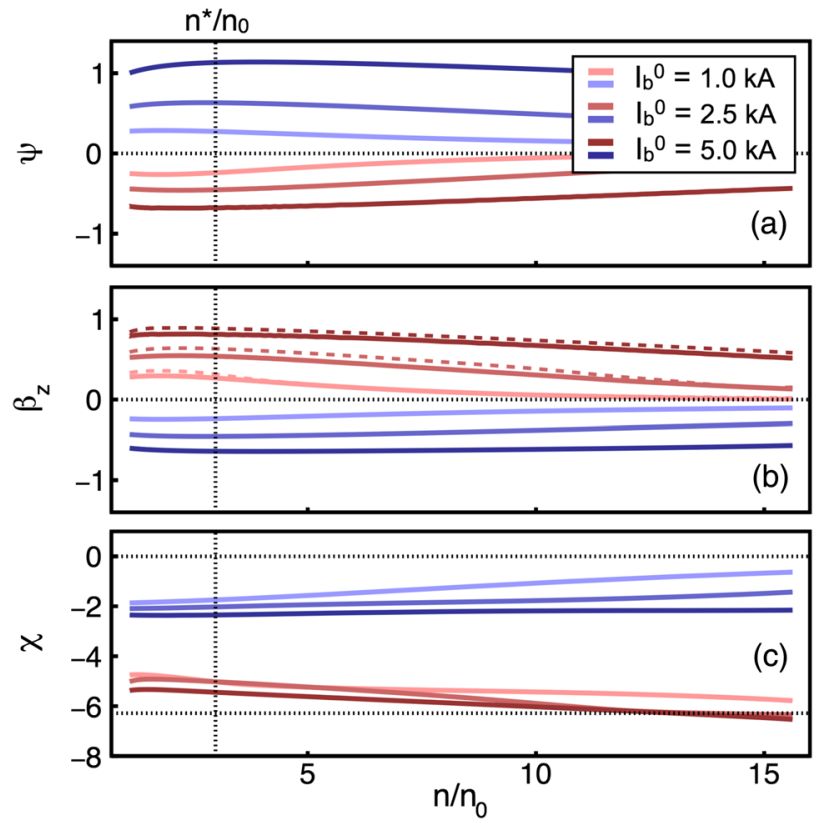

FIG. 5. (a) Maximum (red curves) and minimum (blue curves) values of the (on-axis) wakefield potential $\psi$ as a function of the plasma density, from 3 PIC simulations with drive-beam peak current of $1.0,2.5$, and $5.0 \mathrm{kA}$. (b) Longitudinal velocity of the plasma electrons calculated from the $\psi$ values in panel (a) by means of Eq. (8). Dotted curves show the actual maximum electron velocity $\beta_{z, \max }$ as retrieved from the simulations. (c) Phase-position of the extrema of $\psi$. The red curves show the value of $\chi_{1}$.
Using these values for the extrema of $\psi$, we compute the extrema of $\beta_{z}$ [Fig. 5(b)] by means of Eq. (8), which holds under the QSA, and assuming $\beta_{x}^{2} \ll 1$. In Fig. 5(b) we show the maximum $\beta_{z}$ of the plasma electrons (dotted lines) obtained from the simulations [Fig. 3(d)]. The values of $\beta_{z, \text { max }}$ obtained from simulations are slightly greater than the ones calculated through Eq. (8) (with $\epsilon=1$ ), mainly due to the fact that $\beta_{x}$ is non-negligible at these positions. Figure 5(c) shows the phase-position of the extrema of $\psi$. We observe that, for the given beam parameters, the highest electron velocity is generally reached for density values slightly below $n^{*}$. This defines the density at which it is easiest to trap electrons by means of a DDR. We use the $\beta_{z, \max }$ functions shown in Fig. 5(b) as a measure of the maximum velocity of the electrons after one oscillation, for the different simulated cases. Back to Fig. 1(a), we plot $\beta_{z, \text { max }}$ over the wake phase velocity at $\chi=-2 \pi$, for a Gaussian DDR with $\tilde{n}_{\text {top }}=10$ and $k_{p}^{0} \sigma_{l}=2.5$. The minimum phase velocity that can be reached during a Gaussian DDR, during the first plasma oscillation, is plotted in Fig. 1(b) as a function of the height and the length of the ramp. In addition, we draw the contours for the overall maximum longitudinal velocity of the electrons for the three simulations with $I_{b}^{0}=1.0,2.5$ and $5.0 \mathrm{kA}$. In order to ensure trapping from a DDR the height and the length of the ramp must be such that $\beta_{z, \max }>\beta_{\mathrm{ph} \text {,min }}$ [Fig. 1(b)]. In absolute terms, trapping is easiest when the plasma density at which the phase velocity is minimum complies with the density at which the plasma electrons are fastest in forward direction [cf. Fig. 5(b)]. Therefore, those velocities in Fig. 1(b) smaller than $\beta_{z, \text { max }}$ define a region in the parameter space of the ramp for which trapping is guaranteed.

\section{SIMULATION SETUP AND RESULTS}

In this section we analyze results from three-dimensional PIC simulations, performed with the code OSIRIS [22], where witness bunches are injected by means of the DDR method. The drive-beam parameters adopted in the simulations are taken from the FLASHForward project [18], which aims to realize experimentally this injection method in PWFAs. The drive-beams are hereby supplied from the FLASH accelerator at DESY, with the following characteristic parameters: $1 \mathrm{GeV}$ energy beams with $0.1 \%$ relative energy spread, $\sigma_{z}=25 \mu \mathrm{m}$ rms length, $\sigma_{x, y}=6 \mu \mathrm{m} \mathrm{rms}$ width, $2 \mu \mathrm{m}$ normalized transverse emittance and a peak current of $I_{b}^{0}=2.4 \mathrm{kA}$. In the simulations, the beams are approximated by bi-Gaussian distributions in both longitudinal and transverse directions. The total charge of the beam is $Q=\sqrt{2 \pi} \sigma_{z} c I_{b}^{0}=500 \mathrm{pC}$. From the rms length of the driver, we define the resonant plasma density as $k_{p}^{*} \sigma_{z}=\sqrt{2}$, which for the FLASHForward case gives $n^{*}=0.9 \times 10^{17} \mathrm{~cm}^{-3}$. As we discussed in the previous section, we expect that the plasma electrons are fastest when the drive-beam operates at this density [see Fig. 5(b)]. 
This maximum longitudinal velocity is $\beta_{z, \max } \simeq 0.64$, for $2.5 \mathrm{kA}$ peak current beams. In order to trap plasma electrons along a Gaussian DDR, by employing these drive-beams, the ramps must be sufficiently high and short, such that the minimum phase velocity of the wakefields after one oscillation, $\beta_{\mathrm{ph} \text {,min }}$ is smaller than $\beta_{z, \max }$ [cf. Fig. 1(a)]. From Fig. 1(b) we see that, for $2.5 \mathrm{kA}$ peak current driver beams, the latter condition is fulfilled for Gaussian ramps of $\tilde{n}_{\text {top }} \gtrsim 10$ and $\tilde{\sigma}_{l} \lesssim 2.5$. For this reason, in this simulation study, we pick a Gaussian DDR case with these parameters as reference, namely $\tilde{n}_{\text {top }}=10$ and $\tilde{\sigma}_{l}=2.5$. In Fig. 2 we show that when $\tilde{n}_{\mathrm{top}}=10, \beta_{\mathrm{ph}, \min }$ is reached at a distance $z_{\min } / \sigma_{l} \approx$ 2 with respect to the beginning of the ramp (the top part), which we make coincide with the resonant plasma density for the given driver. The latter condition can be expressed mathematically as $\tilde{n}^{*}=\tilde{n}\left(z_{\min }\right)$, which yields $\tilde{n}^{*}=2.22$, and therefore $n_{0}=0.4 \times 10^{17} \mathrm{~cm}^{-3}$, and $\left(k_{p}^{0}\right)^{-1}=26.6 \mu \mathrm{m}$. Using these parameters for the driver and the ramp, a first 3D simulation was conducted by employing a moving box with the longitudinal and transverse sizes $11 \times 8 \times 8\left(k_{p}^{0}\right)^{-1}$ and resolution $0.020 \times 0.032 \times 0.032\left(k_{p}^{0}\right)^{-1}$, while 4 macroparticles per cell were used to describe both plasma and drivebeam electron species. In order to reduce numerical Cherenkov radiation (NCR) affecting the phase-space of the injected bunches, we have used a NCR-suppressing field solver [29]. The injection process of a witness bunch consisting of electrons from the background plasma can be clearly observed in Fig. 6, where we show the plasma electron density distribution at four different positions along the DDR. In addition to this case, we have performed two more simulations for which we increase the length of the Gaussian-DDR $\left(\tilde{\sigma}_{l}=5.0\right.$ and $\left.\tilde{\sigma}_{l}=7.5\right)$, and another two where we change the top value of the density with respect to the plateau $\left(\tilde{n}_{\text {top }}=5\right.$ and $\tilde{n}_{\text {top }}=2.5$ ), while keeping the other parameters constant. For these last two cases in which we changed $\tilde{n}_{\text {top }}$, we also needed to change $n_{0}$ in order to make the

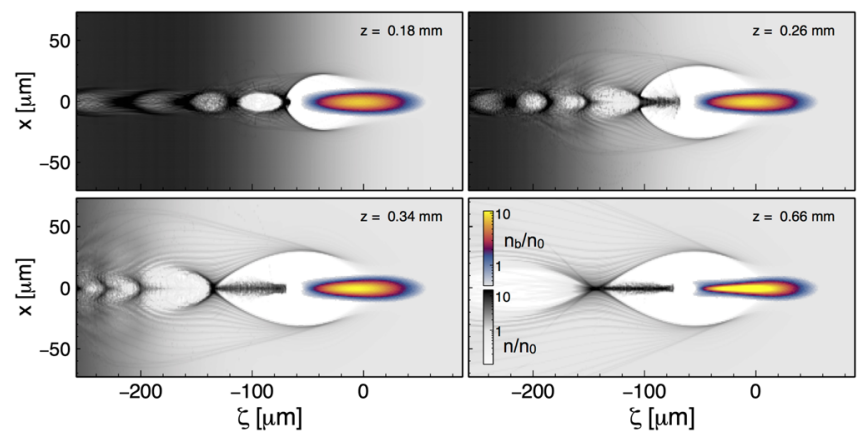

FIG. 6. Plasma and drive-beam densities along the density down-ramp region in a 3D PIC simulation (central horizontal slice of the simulation). $\tilde{n}_{\text {top }}=10$ and $\tilde{\sigma}_{l}=2.5$ in this case.

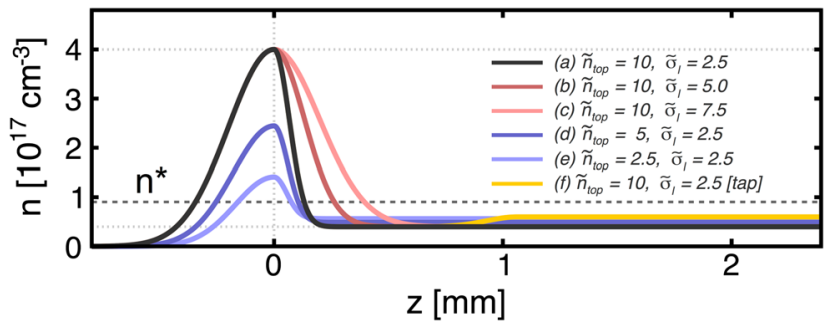

FIG. 7. Different plasma density profiles considered in the PIC simulations.

resonant density to comply with the value of the density at $z_{\text {min }}$, which for $\tilde{n}_{\text {top }}=5$ is approximately $z_{\min } \simeq 1.8 \sigma_{l}$ and for $\tilde{n}_{\text {top }}=2.5, z_{\text {min }} \simeq 1.4 \sigma_{l}$. (cf. Fig. 2), and therefore, $n_{0}=0.5 \times 10^{17} \mathrm{~cm}^{-3}$ and $0.58 \times 10^{17} \mathrm{~cm}^{-3}$, respectively. In addition, we show the result of a sixth simulation with the same ramp parameters as the reference case $\left(\tilde{n}_{\text {top }}=10\right.$ and $\left.\tilde{\sigma}_{l}=2.5\right)$, but in which the plateau plasma density is increased by a factor 1.5 after injection (tapered case). The different plasma density profiles considered in this series of simulations are summarized in Fig. 7. Figure 8 shows the witness beams properties for each of the simulations described above, after a propagation distance of $5 \mathrm{~cm}$ with respect to the beginning of the ramp.

\section{A. Reference case}

For the reference case (a), the injected bunch has total charge of $\sim 140 \mathrm{pC}$, and an approximately flat-top current profile of $0.9 \mathrm{kA}$ and $50 \mu \mathrm{m}$ long $(14 \mu \mathrm{mrms})$. The energy gain after $5 \mathrm{~cm}$ of propagation in the plasma wake is $\left\langle\Delta p_{z, \mathrm{w}}\right\rangle=0.360 \mathrm{GeV}$ on average, with $20 \%$ total energy spread. The latter is the result of the characteristic slope of $E_{z}$ along the bunch, which is related to the correlated relative energy spread of the witness beam, given by $\delta p_{z \text {,corr }}=\left\langle z p_{z}\right\rangle /\left\langle z^{2}\right\rangle\left\langle p_{z}\right\rangle=-1.45 \% / \mu \mathrm{m}$. At this point of the propagation, the maximum energy loss in the driver beam is $\Delta p_{z, d, \max }=-0.233 \mathrm{GeV}$. In the context of this analysis we define the ratio of the average energy gain of the witness beam over the maximum energy loss of the drive-beam as the average transformer ratio, which is $\langle R\rangle \equiv\left|\left\langle\Delta p_{z, \mathrm{w}}\right\rangle / \Delta p_{z, \mathrm{~d}, \max }\right| \simeq 1.5$ in this case. Figure 8(a) shows the longitudinal phase space $\left(p_{z}\right.$ vs $\zeta$ ) (top panel), and the sliced uncorrelated properties (bottom panel) of the injected bunch. The sliced energy spread of the witness beam around its center is $\sim 0.3 \%$, while the sliced normalized transverse emittance is found to be below $0.3 \mu \mathrm{m}$, on average. Small modulations visible in the longitudinal phase space distribution are owed to spurious numerical field errors. However, the usage of a NCR-suppressing field solver greatly improves the consistency of the results when compared to previous results which employed a standard finite-difference timedomain (Yee) field solver [19]. 

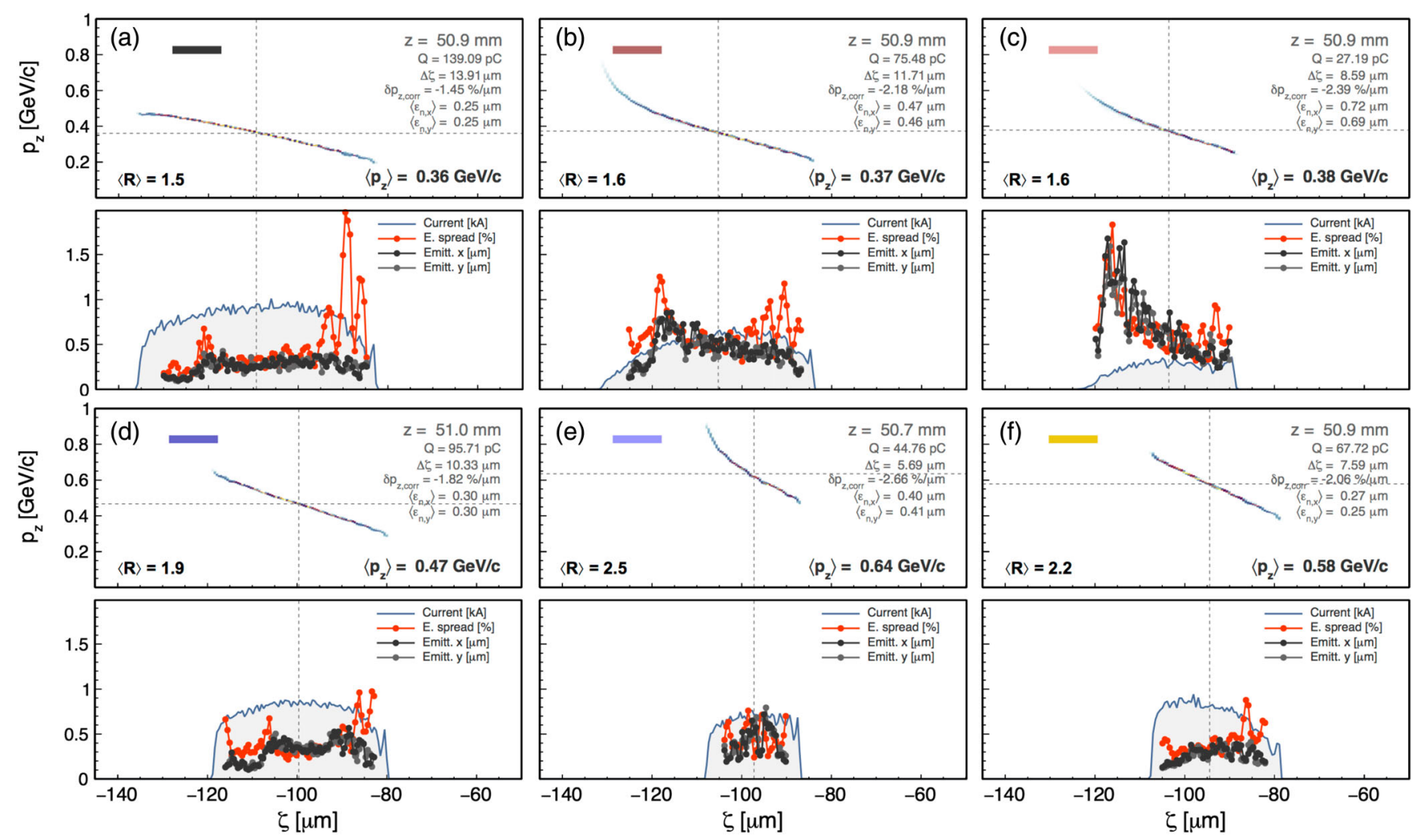

FIG. 8. (Top) Longitudinal phase-space and (bottom) sliced energy spread, normalized transverse emittance and peak current of the witness bunches after a propagation distance of $\sim 5 \mathrm{~cm}$. In these simulations, only the plasma density profile is varied. See Fig. 7 for a graphical representation of the considered profiles.

\section{B. Longer ramps}

With respect to the case (a), the amount of injected charge diminishes drastically when the length of the ramp is increased, featuring $\sim 75 \mathrm{pC}$ for the case (b), and $\sim 27 \mathrm{pC}$ for the case (c), as expected from the increase of the phase velocity $\beta_{\mathrm{ph} \text {,min }}$ for longer ramps Eq. (2). The length of the bunches also decreases, but in lesser degree, obtaining $\sim 12 \mu \mathrm{m}$ (rms) and $\sim 9 \mu \mathrm{m}$ (rms) for the cases (b) and (c), respectively. As a consequence, bunches (b) and (c) feature substantially less current: $\sim 0.5 \mathrm{kA}$ and $\sim 0.3 \mathrm{kA}$, respectively. Bunches with sufficiently high current can partially compensate the slope of the accelerating field through beam-loading $[9,30]$. Therefore, the magnitude of the correlated energy spread (energy chirp) of the bunch is expected to decrease when the injected current increases. This can be clearly observed by comparing the cases (a), (b), and (c) in Fig. 8, with $\delta p_{z, \text { corr }}=-1.45,-2.18$, and $-2.39 \% / \mu \mathrm{m}$, respectively. Due to beam loading, also the average energy gain of the witness diminishes when increasing the charge. Such is also seen in Fig. 8, but the effect is relatively small, and the average transformer ratio remains essentially the same. Other important properties as the average slice emittance and relative energy spread also increase when increasing the length of the ramp (this effect is further discussed in the next section).

\section{Lower ramps}

For the cases (d) and (e) the height of the ramp was decreased to $\tilde{n}_{\text {top }}=5$ and 2.5 , respectively. Besides, the plateau density is increased with respect to (a) in order to keep the resonant density complying with the value of the density at $z_{\min }$. The most obvious change to observe is a decrease of the amount of injected charge, featuring $\sim 96 \mathrm{pC}$ for the case (d), and $\sim 45 \mathrm{pC}$ for the case (e) [Figs. 8(d) and (e)]. In these cases, the decrease in charge is more related to the shortening of the bunches, $\sim 10 \mu \mathrm{m}$ (rms) for case (d) and $\sim 6 \mu \mathrm{m}$ (rms) for case (e), due to the smaller differences in density along the ramp [Eq. (6)]. This effect is also expected from the initial theoretical considerations in Sec. II, where we saw that increasing the ramp length has a stronger effect in diminishing the minimum phase velocity of the wake than decreasing the ramp height [Fig. 1(b)]. The electric current for these cases is therefore more similar to case (a): $\sim 0.8 \mathrm{kA}$ for case (d) and $\sim 0.7 \mathrm{kA}$ for case (e). Provided that the bunches are shorter, they are (on average) closer to the end of the accelerating cavity, and therefore, they are accelerated at a higher transformer ratio: $\langle R\rangle \simeq 1.9$ for case (d) and $\langle R\rangle \simeq 2.5$ for case (e). However, there is an additional increase of the correlated energy spread [similarly to what happened with case (d)]. On one hand, since 
the final plasma density is higher than in case (a), and $\delta p_{z, \text { corr }}=\partial_{z} E_{z} / E_{z} \propto \sqrt{n_{0}}$, we expect an increase of the slope of the accelerating field. On the other hand, the current required to compensate the accelerating field slope is higher for higher transformer ratio cases in PWFAs [9], and therefore, the beam-loading effects are reduced in this case, leading to an overall higher correlated energy spread: $\delta p_{z, \text { corr }}=-1.82 \% / \mu \mathrm{m}$ for case (d) and $\delta p_{z, \text { corr }}=$ $-2.66 \% / \mu \mathrm{m}$ for case (e). Decreasing the height of the ramp also influences the uncorrelated energy spread and emittance, but the effect is not as pronounced as when the length of the ramp is increased. For the three cases together [(a), (d), and (e)], the average slice emittance and the relative energy spread are below $0.5 \mu \mathrm{m}$ and $0.5 \%$, respectively.

\section{Plasma tapered case}

In the simulation case (f), the plasma density is increased by a factor 1.5 shortly after a DDR identical to case (a). As a result, the witness bunch is cut in half (approximately) due to the rephasing of the rear electrons into the second plasma oscillation bucket, by virtue of Eq. (4). The total charge of the witness beam for the case (f), after the plasma taper, is around $70 \mathrm{pC}$, and its slice properties (electrical current, uncorrelated energy spread, and emittance) remain essentially the same as in case (a). Due to the increase in plasma density and the rephasing, this bunch is accelerated at a higher rate. After $\sim 5 \mathrm{~cm}$ the average energy of the witness is $0.580 \mathrm{GeV}$, while the maximum energy loss in the driver beam is $0.263 \mathrm{GeV}$, resulting in an average transformer ratio of $\langle R\rangle=2.2$, largely increased with respect to case (a). However due to the fact that the slope in the accelerating field scales as the square root of the plasma density, the correlated average energy spread is greater in this case $\delta p_{z, \text { corr }}=-2.06 \% / \mu \mathrm{m}$.

In Fig. 9 we show the evolution of the average energy (top), the correlated relative energy spread (middle), and the average slice emittance (bottom) of the injected witness bunches, during the first $5 \mathrm{~cm}$ of acceleration for the selected simulation cases. From these results we observe that the most interesting witness beams are obtained when employing shorter DDRs. For these cases [(a), (d), and (e)], the average sliced emittance and the correlated energy spread are smaller, at the same time the injected current is higher. On the other hand, we observe an increase of the acceleration performance when the height of the ramp is reduced, due to the fact that the injected electron bunches are shorter and closer to the end of the first oscillation bucket, where the accelerating field is highest. Therefore, we conclude that shorter and lower ramps are convenient for the production of witness bunches with optimized properties, i.e., high current, low energy spread and emittance, and high transformer ratio. Tapering the final plasma density to a higher value [case (f)], it is shown to be an effective way of increasing the transformer ratio while keeping the slice properties of the bunch.

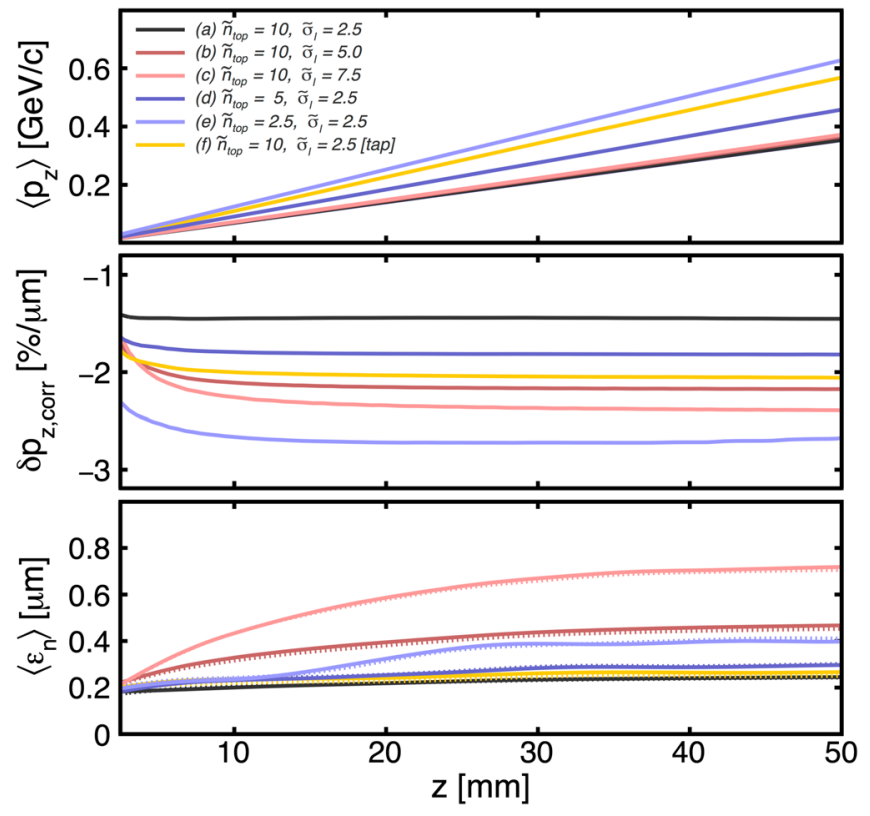

FIG. 9. (Top) Average longitudinal momentum, (middle) correlated relative energy spread and (bottom) average slice emittance of the witness bunches as a function of acceleration distance for the selected simulation cases.

\section{EMITTANCE REDUCTION BY CHARGE INJECTION}

While the variation of injected charge, the bunch duration, the acceleration rate and the correlated energy spread of the injected bunches can be explained qualitatively by means of simple theoretical considerations for the DDR injection principle, it is not that straight-forward to show why shorter ramps lead to a reduction of the slice emittance of the beams [see Fig. 9 (bottom)]. In order to describe this effect, we present an analysis on the macroparticle trajectories (in 6D phase-space), for the simulation cases (a) and (b) in Fig. 7, which only differ on the length of the ramp, $\tilde{\sigma}_{l}=2.5$ for case (a) and $\tilde{\sigma}_{l}=5.0$ for case (b). In Fig. 10, we show the trajectories of the electrons coming from (approximately) the same initial density range (from $2.00 n_{0}$ to $2.03 n_{0}$ ), for both cases. Top, middle, and bottom panels show, respectively, the trajectories of these selection of macroparticles in the $r$ versus $\zeta, \beta_{r}$ versus $\zeta$, and $\beta_{z}$ versus $\zeta$ planes. Here $\beta_{r} \equiv \sqrt{p_{x}^{2}+p_{y}^{2}} /(m c \gamma)$. One can see from the figure that the macroparticle trajectories are qualitatively similar for these two cases while the longitudinal velocity is still negative. However, as soon as the electrons start to gain velocity in forward direction, we observe a substantial difference in the trajectories. On one hand, for the electrons in the longer ramp case (b), their longitudinal velocity is increased at a higher rate. Because the phase velocity is higher in this case, the phase shift of these electrons is smaller [Eq. (3)], and therefore, these electrons remain closer to the end of the accelerating cavity 


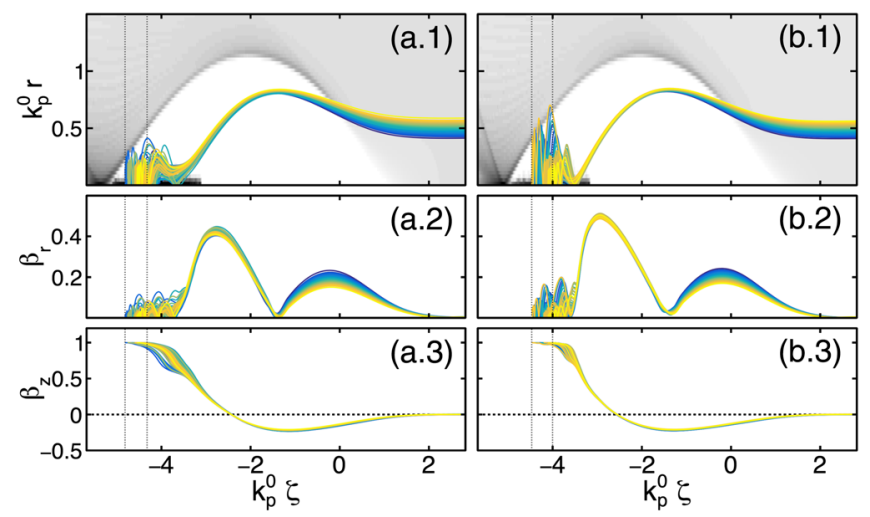

FIG. 10. Trajectory of the macroparticles originated from the same initial plasma density range (from $2.00 n_{0}$ to $2.03 n_{0}$ ) for two different ramp length cases (a) and (b). (Top) Radial distance to the beam axis $r$, (middle) "transverse velocity" $\beta_{r}=p_{r} /(m c \gamma)$, (bottom) longitudinal velocity $\beta_{z}=p_{z} /(m c \gamma)$ versus the comoving variable $\zeta$. In the top panels, we plot for reference the particle distribution of the plasma electrons after the injection process has been terminated.

during the injection process. This means that they reach relativistic velocities $\left(\beta_{z} \approx 1\right)$ earlier, and consequently, their final phase positions $\zeta_{f}$ are advanced with respect to the shorter ramp case (Fig. 10). On the other hand, we observe that for the case (a), the electrons also gain less transverse velocity towards the propagation axis. In Appendix we show that the transverse motion of the electrons inside the blowout can be approximated by a harmonic oscillator, driven by the (linear) restoring force $F_{x}(\zeta, x) \approx-K(\zeta) x$, where $K$ symbolizes the focusing strength. In absence of any beam current $K(\zeta) \simeq$ $m \omega_{p}^{2} \hat{k}(\zeta)$, where $\hat{k}(\zeta)$ is a normalized focusing strength that depends on the particularities of the blowout shape and on the longitudinal velocity of the electrons (cf. Appendix). In the following, we assume that $\hat{k}$ is independent of the plasma density value in the range where injection occurs along the DDR. The amplitude in transverse momentum for a single electron trajectory is then related to the amplitude in the transverse coordinate by $p_{x, 0}=\sqrt{\hat{k}} m c\left(k_{p} x_{0}\right)$. For shorter ramps the plasma density decreases more rapidly and therefore the focusing strength applied to the electrons becomes effectively weaker during the trapping process, resulting in a smaller oscillation amplitude (cf. Fig. 10). In addition, because shorter ramps lead to the trapping of more charge, it is expected that beam loading effects are stronger for case (a). The space-charge field of the injected electrons contributes to further reduce the net focusing strength acting on the electrons during injection, and therefore dumps the oscillation amplitude (cf. Appendix). This effect is stronger as the electrons approach the propagation axis, and can be observed between $-4<k_{p}^{0} \zeta<-3$ in Fig. 10 (a.1), where many of the macro-particle trajectories do not even reach the propagation axis after the first oscillation [in contrast to Fig. 10 (b.1)]. As a result, the final transverse momentum amplitude of the trapped electrons is expected to be lower in the case of shorter ramps [cf. Figs. 10 (a.2) and (b.2)].

Assuming cylindrical symmetry and full betatron decoherence of the electron trajectories, an upper estimate of the normalized transverse emittance $\epsilon_{n, x}=$ $\sqrt{\left\langle x^{2}\right\rangle\left\langle p_{x}^{2}\right\rangle-\left\langle x p_{x}\right\rangle} / m c$, can be given in terms of the variance of the initial radial distribution of the electrons composing the slice and the characteristic focusing strength [31]

$$
\epsilon_{n, x}=\sqrt{\frac{K}{m c^{2}}} \frac{\left\langle r_{i}^{2}\right\rangle}{2},
$$

with $\left\langle r_{i}^{2}\right\rangle=\int r_{i}^{3} f\left(r_{i}\right) \mathrm{d} r_{i} / \int r_{i} f\left(r_{i}\right) \mathrm{d} r_{i}$, and $f\left(r_{i}\right)$ the initial distribution function of the electrons. In Fig. 11 top (middle), we show the initial normalized radius $k_{p} r_{i}$ (initial density $n_{i} / n_{0}$ ) of the injected electrons versus their final phase positions $k_{p}^{0} \zeta_{f}$ for the simulation cases (a) and (b). In general, we can observe a clear correlation between $\zeta_{f}$ and $n_{i}$, but an approximately constant distribution for $k_{p} r_{i}$ as a function of $\zeta_{f}$. In the limit of long ramps, where the rephasing effects are less pronounced, and the system is closer to the quasistatic picture, we may expect that the focusing strength over the electrons getting injected corresponds to the one caused by the blowout at the initial density $K \approx m \omega_{p, i}^{2} \hat{k}$ and therefore, from Eq. (9), $k_{p}^{0} \epsilon_{n, x} \approx$ $\sqrt{\hat{k} / \tilde{n}_{i}}\left\langle\left(k_{p} r_{i}\right)^{2}\right\rangle / 2$. This expression allows us to isolate the dependency of the emittance on the local plasma density, from where the electrons composing a slice are originating $\epsilon_{n, x} \propto \sqrt{\hat{k} / \tilde{n}_{i}}$. Such a correlation between $\epsilon_{n, x}$ and $\tilde{n}_{i}$ can be observed in cases (b) and (c), where the sliced normalized emittance grows towards the back of the

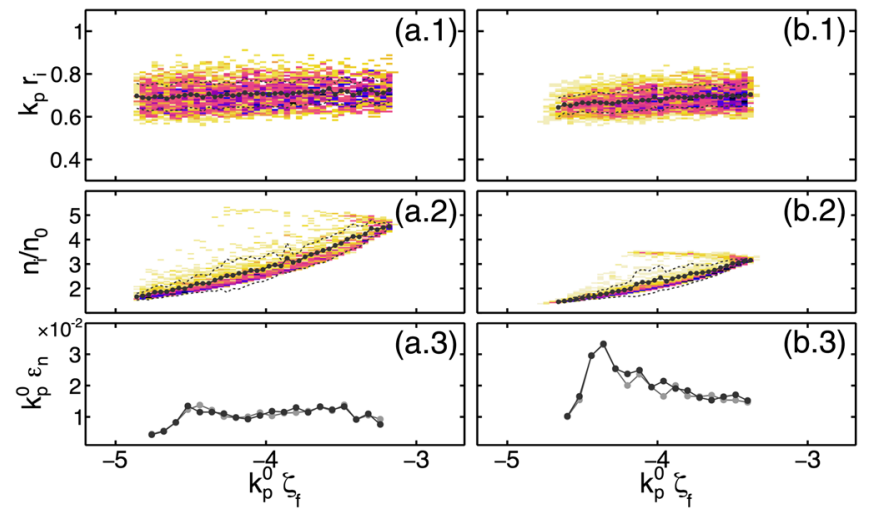

FIG. 11. Initial radius (top) and density (middle) distributions for trapped electrons as a function of their final phase positions, for the cases (a) and (b) in Fig. 7. Black dots indicate the average values and dashed lines $\pm 1 \mathrm{rms}$. Sliced normalized emittance in horizontal (black) and vertical (gray) directions after $\sim 5 \mathrm{~cm}$ of acceleration. 
injected bunch as the initial density diminishes [cf. Fig. 11 (b.3) and Figs. 8(b) and (c)]. However for the case (a), where the ramp length is shorter and more charge was trapped, the sliced emittance of the beam remains flat (and even decreases) towards the end of the bunch. This effect might be explained as follows: On one hand, when the plasma density changes more rapidly toward lower values, the focusing strength diminishes as $K \approx m \omega_{p}^{2} \hat{k}$, with $\omega_{p}$ the local plasma frequency, and therefore, the emittance should be lower. On the other hand, because beam-loading effects are stronger when more charge is trapped when employing shorter ramps, these significantly contribute to further diminish the normalized focusing strength $\hat{k}$ during the trapping process. As a result, we conclude that injecting electrons during a shorter ramp favors the production of higher current beams, which in turn, leads to a lower normalized emittance and a lower projected energy spread due to beam-loading effects.

\section{SUMMARY}

In this work, we have reviewed the principles of the density down-ramp (DDR) injection technique in PWFAs, in order to establish the necessary requirements for injection and acceleration. Trapping of plasma electrons in the first acceleration bucket is possible if the electrons propagate equal to or faster than the phase-velocity of the wake. Assuming that the plasma excitation regime does not change significantly during the DDR, the phase velocity of the wake is essentially defined by the ramp profile [Eq. (2)]. By means of PIC simulations we were able to determine the maximum plasma-electron velocity for three electron-driver cases that differ only on their peak current. This maximum velocity strongly depends on the current of the drive-beam and it is approximately reached at the resonant density for these three cases (Fig. 5). Therefore, in order to facilitate injection, it is useful to match the density at which the phase velocity is minimum to the resonant plasma density of the driver. Based on these considerations, we have determined that, for moderate current drivers ( $2.5 \mathrm{kA})$, ramps of a length smaller than the plasma wavelength (at the plateau density) are required (Fig. 1). Additional PIC simulations have been employed to study and optimize the properties of the injected bunches for different Gaussian-DDR profiles. We find that shorter ramps lead to an increase of the trapped charge, which in turn, contributes to substantially reduce the emittance (Fig. 8). This interesting finding can be explained by the fact that the transverse motion of mildly relativistic plasmaelectrons is damped during the injection process by the space-charge field of the previously injected charge, thereby reducing the emittance. Besides, an increased trapped charge reduce the correlated energy spread of the injected beams through beam loading (Fig. 9). For the case of FLASHForward-type of drive-beams, with
$500 \mathrm{pC}$ of charge and $2.5 \mathrm{kA}$ of peak current, best witness beam properties in terms of charge $(140 \mathrm{pC})$, current $(\sim 1 \mathrm{kA})$, normalized emittance $(\sim 0.25 \mu \mathrm{m})$ and correlated energy spread $(\sim-1.45 \% / \mu \mathrm{m})$, were obtained using a Gaussian-DDR with a top-to-plateau factor of 10 and $\sigma_{l} \approx 66 \mu \mathrm{m}$. As a result of this analysis, we expect that further increasing the injected charge (by employing shorter ramps and/or higher current drivers) can be an effective way for the production of higher current witness beams with lower values of energy spread and emittance.

\section{ACKNOWLEDGMENTS}

We thank the OSIRIS consortium (IST/UCLA) for access to the OSIRIS code. Special thanks go to R. Lehe for support with the usage of his solver. Furthermore, we acknowledge the grant of computing time by the Jülich Supercomputing Center on JUQUEEN under Project No. HHH23 and the use of the High-Performance Cluster (Maxwell) at DESY. T. J.M acknowledges the support by the Deutscher Akademischer Austauschdienst (German Academic Exchange Service) with funds from the Bundesministerium für Bildung und Forschung and the Marie Sklodowska Curie Actions of the EU's FP7 under REA grant no. 605728 (P. R. I. M.E.). Z.H. and A.M. would like to thank, respectively, the China and Germany Postdoctoral Exchange Program and the Helmholtz Virtual Institute VH-VI-503, for financial support.

\section{APPENDIX: TRANSVERSE DYNAMICS OF PLASMA ELECTRONS INSIDE THE BLOWOUT}

Assuming rotational symmetry with respect to the propagation axis of the drive-beam, the transverse force over a plasma electron is given by just one component $F_{x}=-e\left(E_{x}-\beta_{z} c B_{y}\right)$, with $\beta_{z}$ the longitudinal velocity of the electron. The transverse wakefields are linear inside the blowout [10] $\left(E_{x}-c B_{y}\right) / E_{0}=\left(k_{p} x\right) / 2$. The transverse force on an plasma-electron inside the blowout cavity can be written in terms of $E_{x}$ only: $F_{x}=-e\left(E_{x}\left(1-\beta_{z}\right)+\right.$ $\left.\beta_{z} E_{0}\left(k_{p} x\right) / 2\right)$. Integrating the Gauss' equation $(\boldsymbol{\nabla E}=$ $\left.\rho / \epsilon_{0}\right)$, and using that $E_{z}$ is constant along the transverse direction $\left(\partial_{x} E_{z}=0\right)$ in the blowout, it is found that

$$
\frac{E_{x}}{E_{0}}=(1-S) \frac{k_{p} x}{2}-\frac{\hat{\Lambda}_{b}(x)}{k_{p} x},
$$

where $S \equiv \partial_{\zeta} E_{z} / k_{p} E_{0}$ is the normalized slope of the longitudinal electric field, and $\hat{\Lambda}_{b}(x) \equiv k_{p}^{2} \int_{0}^{x} r \mathrm{~d} r \tilde{n}_{b}$, with $\tilde{n}_{b}$ the normalized electron density of a beam present in the blowout (driver or witness). Using Eq. (A1), the equation for the transverse motion of an electron inside the blowout yields 


$$
\frac{\dot{p}_{x}}{m c \omega_{p}}=-\frac{k_{p} x}{2}+\left(1-\beta_{z}\right)\left(S \frac{k_{p} x}{2}+\frac{\hat{\Lambda}_{b}(x)}{k_{p} x}\right),
$$

with $p_{x}=m \gamma \dot{x}$, the transverse momentum of the electron. The first term in Eq. (A2) represents the electrostatic attraction due to the uniform background of ions. The second term is only relevant for nonrelativistic electrons and consists on a linear term proportional to $S$ and a (generally nonlinear) term due to the electrostatic repulsion caused by any electron current present in the blowout. For $x$-positions sufficiently near the axis, or in case of an uniform electron-beam distribution around the axis, we can approximate $\hat{\Lambda}_{b}(x) /\left(k_{p} x\right) \simeq \tilde{n}_{b}^{0} k_{p} x / 2$, with $\tilde{n}_{b}^{0}$ the (normalized) peak electron density of a beam centered on the propagation axis. Further assuming that $\dot{p}_{x} \simeq m \gamma \ddot{x}$, Eq. (A2) can be simplified to $\ddot{x}=-\omega_{p}^{2} \hat{k} x$, the equation for a harmonic oscillator with restoring force $F_{x}=-K x=$ $-m \omega_{p}^{2} \hat{k} x$, where

$$
\hat{k}=\frac{1}{2 \gamma}\left[1-\left(1-\beta_{z}\right)\left(S+\tilde{n}_{b}^{0}\right)\right],
$$

represent the (normalized) focusing strength over the electrons. In general $\hat{k}$ depends on the $\zeta$-position of the electron, and therefore, the amplitude and the frequency of the oscillation changes along the electron orbit inside the blowout. It can be shown that, for high-current electron drivers $\left(\Lambda_{b} \gg 1\right), S \rightarrow 1 / 2$ in the central region of the blowout [11]. For moderate current drivers (in the range of few kA) [9] $S \approx 0.2$. The value of $S$ increases substantially at the rear of the blowout cavity, but also the longitudinal velocity of the plasma electrons increases significantly and the influence of a high $S$ is partially balanced by a small $\left(1-\beta_{z}\right)$ term in Eq. (A3). In order to quantify this effect we plot in Fig. $12 E_{z} / E_{0}, S, \beta_{z}$ and $\hat{k}$

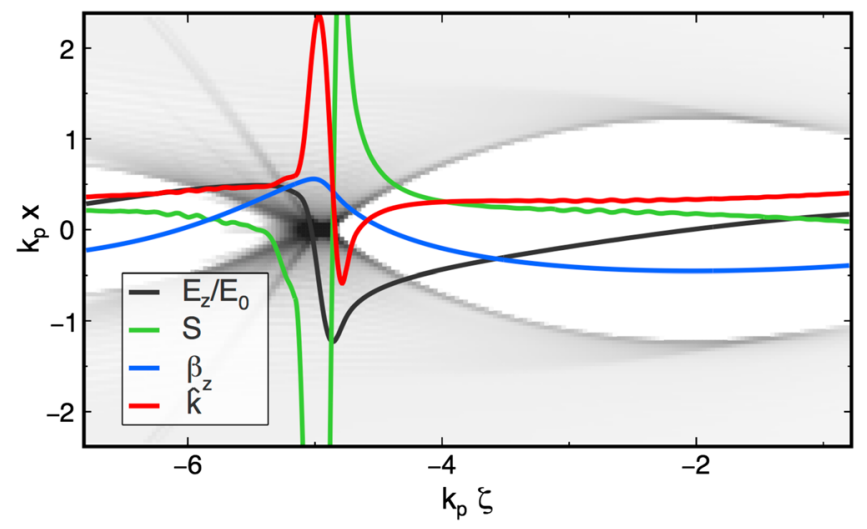

FIG. 12. Plasma electron density in the blowout regime from a PIC simulation with a $2.5 \mathrm{kA}$ electron driver (grey scale). The (normalized) longitudinal electric field $E_{z} / E_{0}$ (black), its normalized derivative $S$ (green), the longitudinal velocity of the plasma electrons $\beta_{z}$ (blue), and the normalized focusing strength over the electrons $\hat{k}$ (red). as obtained from the PIC simulation shown in Fig. 3, for a $2.5 \mathrm{kA}$ driver. Here $\beta_{z}$ is calculated from the value of $\psi$ in the propagation axis through Eq. (8), that we use to estimate $\hat{k}$ from Eq. (A3). We observe that $\hat{k} \approx 0.3$ for electrons going through most of the central region of the blowout. When the electrons approach the vertex of the blowout, the focusing strength suddenly decreases to $\hat{k} \approx-0.5$. This reduction of $\hat{k}$ at the end of the blowout damps the oscillation amplitude of the plasma electrons. In addition, in the presence beam charge near the vertex of the blowout, the oscillation amplitude of the electrons is further damped by the $\hat{\Lambda}_{b} /\left(k_{p} x\right)$ term in Eq. (A2), or the term $\tilde{n}_{b}^{0}$ in Eq. (A3). If after completing their first oscillation the electrons are subsequently trapped in the blowout (e.g., by means of a DDR), the focusing strength increases again to $\hat{k}=1 /(2 \gamma)$ when $\beta_{z} \rightarrow 1$ [Eq. (A3)], and the frequency of their oscillations (betatron frequency) is given by $\omega_{\beta}=\omega_{p} / \sqrt{2 \gamma}$.

[1] V. Veksler, Coherent principle of acceleration of charged particles, Symposium du CERN sur les Accélérateurs de Haute Energie et la Physique des Mesons $\pi$ (CERN, Geneva, 1956), Vol. 1, 80.

[2] P. Chen, J. M. Dawson, R. W. Huff, and T. Katsouleas, Acceleration of Electrons by the Interaction of a Bunched Electron Beam with a Plasma, Phys. Rev. Lett. 54, 693 (1985).

[3] I. Blumenfeld, C. E. Clayton, F.-J. Decker, M. J. Hogan, C. Huang, R. Ischebeck, R. Iverson, C. Joshi, T. Katsouleas, N. Kirby, W. Lu, K. A. Marsh, W. B. Mori, P. Muggli, E. Oz, R. H. Siemann, D. Walz, and M. Zhou, Energy doubling of $42 \mathrm{GeV}$ electrons in a metre-scale plasma wakefield accelerator, Nature (London) 445, 741 (2007).

[4] M. Litos, E. Adli, J. M. Allen, W. An, C. I. Clarke, S. Corde, C. E. Clayton, J. Frederico, S. J. Gessner, S.Z. Green, M. J. Hogan, C. Joshi, W. Lu, K. A. Marsh, W. B. Mori, M. Schmeltz, N. Vafaei-Najafabadi, and V. Yakimenko, $9 \mathrm{GeV}$ energy gain in a beam-driven plasma wakefield accelerator, Plasma Phys. Controlled Fusion 58, 034017 (2016).

[5] E. Oz, S. Deng, T. Katsouleas, P. Muggli, C. D. Barnes, I. Blumenfeld, F. J. Decker, P. Emma, M. J. Hogan, R. Ischebeck, R. H. Iverson, N. Kirby, P. Krejcik, C. O'Connell, R. H. Siemann, D. Walz, D. Auerbach, C. E. Clayton, C. Huang, D. K. Johnson, C. Joshi, W. Lu, K. A. Marsh, W. B. Mori, and M. Zhou, Ionization-Induced Electron Trapping in Ultrarelativistic Plasma Wakes, Phys. Rev. Lett. 98, 084801 (2007).

[6] B. Hidding, G. Pretzler, J. B. Rosenzweig, T. Königstein, D. Schiller, and D. L. Bruhwiler, Ultracold Electron Bunch Generation via Plasma Photocathode Emission and Acceleration in a Beam-Driven Plasma Blowout, Phys. Rev. Lett. 108, 035001 (2012).

[7] F. Li, J. F. Hua, X. L. Xu, C. J. Zhang, L. X. Yan, Y. C. Du, W. H. Huang, H. B. Chen, C. X. Tang, W. Lu, C. Joshi, W. B. Mori, and Y. Q. Gu, Generation of High Brightness 
Electron Beams via Ionization Induced Injection by Transverse Colliding Lasers in a Beam-Driven Plasma Wakefield Accelerator, Phys. Rev. Lett. 111, 015003 (2013).

[8] A. Martinez de la Ossa, J. Grebenyuk, T. Mehrling, L. Schaper, and J. Osterhoff, High-Quality Electron Beams from Beam-Driven Plasma Accelerators by WakefieldInduced Ionization Injection, Phys. Rev. Lett. 111, 245003 (2013).

[9] A. Martinez de la Ossa, T. J. Mehrling, L. Schaper, M. J. V. Streeter, and J. Osterhoff, Wakefield-induced ionization injection in beam-driven plasma accelerators, Phys. Plasmas 22, 093107 (2015).

[10] J. B. Rosenzweig, B. Breizman, T. C. Katsouleas, and J. J. $\mathrm{Su}$, Acceleration and focusing of electrons in twodimensional nonlinear plasma wake fields, Phys. Rev. A 44, R6189 (1991).

[11] K. Lotov, Blowout regimes of plasma wakefield acceleration, Phys. Rev. E 69, 046405 (2004).

[12] H. Suk, N. Barov, J. B. Rosenzweig, and E. Esarey, Plasma Electron Trapping and Acceleration in a Plasma Wake Field Using a Density Transition, Phys. Rev. Lett. 86, 1011 (2001).

[13] G. Fubiani, E. Esarey, C. B. Schroeder, and W. P. Leemans, Improvement of electron beam quality in optical injection schemes using negative plasma density gradients, Phys. Rev. E 73, 026402 (2006).

[14] K. Schmid, A. Buck, C. M. S. Sears, J. M. Mikhailova, R. Tautz, D. Herrmann, M. Geissler, F. Krausz, and L. Veisz, Density-transition based electron injector for laser driven wakefield accelerators, Phys. Rev. ST Accel. Beams 13, 091301 (2010).

[15] J. Faure, C. Rechatin, O. Lundh, L. Ammoura, and V. Malka, Injection and acceleration of quasimonoenergetic relativistic electron beams using density gradients at the edges of a plasma channel, Phys. Plasmas 17, 083107 (2010).

[16] A. Buck, J. Wenz, J. Xu, K. Khrennikov, K. Schmid, M. Heigoldt, J. M. Mikhailova, M. Geissler, B. Shen, F. Krausz, S. Karsch, and L. Veisz, Shock-Front Injector for High-Quality Laser-Plasma Acceleration, Phys. Rev. Lett. 110, 185006 (2013).

[17] M. Hansson, B. Aurand, X. Davoine, H. Ekerfelt, K. Svensson, A. Persson, C.-G. Wahlström, and O. Lundh, Down-ramp injection and independently controlled acceleration of electrons in a tailored laser wakefield accelerator, Phys. Rev. ST Accel. Beams 18, 071303 (2015).

[18] A. Aschikhin et al., The FLASHForward facility at DESY, Nucl. Instrum. Methods Phys. Res., Sect. A 806, 175 (2016).

[19] J. Grebenyuk, A. Martinez de la Ossa, T. Mehrling, and J. Osterhoff, Beam-driven plasma-based acceleration of electrons with density down-ramp injection at FLASHForward, Nucl. Instrum. Methods Phys. Res., Sect. A 740, 246 (2014).

[20] L. Schaper, L. Goldberg, T. Kleinwächter, J.-P. Schwinkendorf, and J. Osterhoff, Longitudinal gas-density profilometry for plasma-wakefield acceleration targets, Nucl. Instrum. Methods Phys. Res., Sect. A 740, 208 (2014).
[21] G. Wittig, O. Karger, A. Knetsch, Y. Xi, A. Deng, J. B. Rosenzweig, D. L. Bruhwiler, J. Smith, G. G. Manahan, Z.-M. Sheng, D. A. Jaroszynski, and B. Hidding, Optical plasma torch electron bunch generation in plasma wakefield accelerators, Phys. Rev. ST Accel. Beams 18, 081304 (2015).

[22] R. Fonseca, L. Silva, F. Tsung, V. Decyk, W. Lu, C. Ren, W. Mori, S. Deng, S. Lee, T. Katsouleas, and J. Adam, Notes Comp. Sci. 2331, 342 (2002); R. A. Fonseca, S. F. Martins, L. O. Silva, J. W. Tonge, F. S. Tsung, and W. B. Mori, One-to-one direct modeling of experiments and astrophysical scenarios: pushing the envelope on kinetic plasma simulations, Plasma Phys. Controlled Fusion 50, 124034 (2008); R. A. Fonseca, J. Vieira, F. Fiuza, A. Davidson, F. S. Tsung, W. B. Mori, and L. O. Silva, Exploiting multi-scale parallelism for large scale numerical modelling of laser wakefield accelerators, Plasma Phys. Controlled Fusion 55, 124011 (2013).

[23] O. Kononenko, N. Lopes, J. Cole, C. Kamperidis, S. Mangles, Z. Najmudin, J. Osterhoff, K. Poder, D. Rusby, D. Symes, J. Warwick, J. Wood, and C. Palmer, 2D hydrodynamic simulations of a variable length gas target for density down-ramp injection of electrons into a laser wakefield accelerator, Nucl. Instrum. Methods Phys. Res., Sect. A 829, 125 (2016).

[24] P. Mora and J. Thomas M. Antonsen, Kinetic modeling of intense, short laser pulses propagating in tenuous plasmas, Phys. Plasmas 4, 217 (1997).

[25] P. Sprangle, E. Esarey, and A. Ting, Nonlinear Theory of Intense Laser-Plasma Interactions, Phys. Rev. Lett. 64, 2011 (1990).

[26] W. Lu, C. Huang, M. Zhou, W. B. Mori, and T. Katsouleas, Nonlinear Theory for Relativistic Plasma Wakefields in the Blowout Regime, Phys. Rev. Lett. 96, 165002 (2006).

[27] S. A. Yi, V. Khudik, C. Siemon, and G. Shvets, Analytic model of electromagnetic fields around a plasma bubble in the blow-out regime, Phys. Plasmas 20 (2013).

[28] W. Lu, C. Huang, M. M. Zhou, W. B. Mori, and T. Katsouleas, Limits of linear plasma wakefield theory for electron or positron beams, Phys. Plasmas 12, 063101 (2005).

[29] R. Lehe, A. Lifschitz, C. Thaury, V. Malka, and X. Davoine, Numerical growth of emittance in simulations of laser-wakefield acceleration, Phys. Rev. ST Accel. Beams 16, 021301 (2013).

[30] M. Tzoufras, W. Lu, F. S. Tsung, C. Huang, W. B. Mori, T. Katsouleas, J. Vieira, R. A. Fonseca, and L. O. Silva, Beam Loading in the Nonlinear Regime of Plasma-Based Acceleration, Phys. Rev. Lett. 101, 145002 (2008).

[31] N. Kirby, I. Blumenfeld, C. E. Clayton, F. J. Decker, M. J. Hogan, C. Huang, R. Ischebeck, R. H. Iverson, C. Joshi, T. Katsouleas, W. Lu, K. A. Marsh, S. F. Martins, W. B. Mori, P. Muggli, E. Oz, R. H. Siemann, D. R. Walz, and M. Zhou, Transverse emittance and current of multi-GeV trapped electrons in a plasma wakefield accelerator, Phys. Rev. ST Accel. Beams 12, 051302 (2009). 